\title{
Missbrauch und Abhängigkeit von Benzodiazepinen und Z-Drugs
}

\author{
Klinik und Therapie
}

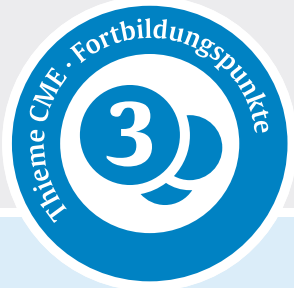

Michael Soyka

Psychiatrische Klinik München, Universität München

\section{ZUSAMMENFASSUNG}

Missbrauch und Abhängigkeit von Sedativa und Hypnotika sind klinisch häufig (Prävalenz ca. 2 \%), insbesondere von Benzodiazepinen und Non-Benzodiazepin-Hypnotika (Z-Drugs). Beide Substanzgruppen haben ältere und weit toxischere Sedativa und Hypnotika wie Barbiturate und Meprobamat zu Recht verdrängt. Benzodiazepine wie Z-Drugs entfalten ihre Wirkung über den inhibitorischen GABA-Rezeptor und können beide eine erhebliche Toleranz induzieren, was klinisch zu Dosissteigerungen, physischer und psychischer Abhängigkeit, Kontrollverlust sowie Entzugssymptomen führen kann. Prädisponierende Faktoren sind neben dem Geschlecht, Frauen sind häufiger betroffen, vor allem psychiatrische und psychosomatische Erkrankungen, insbesondere Angst und Schlafstörun- gen, aber auch psychosomatische Störungen und chronische Schmerzerkrankungen. Besonders häufig und lange werden Benzodiazepine älteren Patienten verschrieben, entgegen aller Leitlinienempfehlungen.

Therapeutisch gesichert ist, dass bei Benzodiazepinabhängigkeit ein langsames Ausschleichen über viele Wochen, manchmal sogar Monate notwendig ist. Ansonsten können erhebliche Entzugserscheinungen inklusive epileptischen Anfällen, Psychosen und Delire auftreten. Das Entzugssyndrom bei Sedativa und Hypnotika ist sehr vielgestaltig mit Depression, Agitation, innerer Unruhe und Perzeptions- und Schlafstörungen als häufigen Symptomen. Die übrige Therapie ist pragmatisch und richtet sich nach den zugrunde liegenden psychischen Störungen und Entzugssymptomen. Im Wesentlichen werden Antidepressiva eingesetzt. Kurzzeitinterventionen werden empfohlen. Psychotherapeutisch haben sich Psychoedukation, kognitive Verhaltenstherapie oder motivationale Therapien bewährt.

\section{Einleitung}

Vor Kurzem wurde unter dem etwas irreführenden Titel „Medikamentenbezogene Störungen“ die erste S3-Leitlinie zu Diagnostik und Therapie der Medikamentenabhängigkeit vorgestellt (AWMF-Register 038-025), die klinisch sehr wichtig ist, aber noch keine weite Verbreitung gefunden hat. Neben vielen anderen Themen (Opioide, Gabapentoide) kommt dem Thema Sedativa und Hypnotika dabei eine große Rolle zu. Vor diesem Hintergrund erscheint es von Interesse, den heutigen Wissensstand zum Thema Abhängigkeit von Benzodiazepinen (BZD) und NonBZD-Hypnotika (Z-Drugs) darzustellen. Sedativa und Hypnotika sind eine sehr heterogene Gruppe von Substanzen, wobei dieser Artikel den Schwerpunkt auf die am häufigsten eingesetzten Substanzen, nämlich Benzodiazepinen und Z-Drugs, setzt. Andere Substanzen wie Barbiturate, Chloralhydrat, Clomethiazol und Meprobamat sind zugunsten diese beiden Substanzgruppen weitestgehend verlassen worden [1-3]. Benzodiazepine werden seit den frühen 1960er-Jahren eingesetzt - nicht immer leitliniengerecht oder exklusiv bei psychiatrischen Erkrankungen wie schon ein Rolling-Stones-Titel aus dem Jahr 1966 „Mother's little Helpers" widerspiegelt:

„Mother needs something today to calm her down and though she's not really ill. There's a little yellow pill. She goes running for the shelter of a mother's little helper. And it helps her on her way, gets her through her busy day ...,

\section{Klinische Einsatzgebiete von Benzodiazepinen und Z-Drugs}

Z-Drugs werden per Definition zur Behandlung von Schlafstörungen (Insomnie) eingesetzt. Benzodiazepine werden, etwas willkürlich vor allem aufgrund ihrer Wirkdauer, in Hypnotika/Sedativa und Anxiolytika unterschieden. Anxiolytika und Tranquilizer werden zur Behandlung von Angststörungen, innere Unruhe oder bei verschiedenen psychiatrischen Erkrankungen eingesetzt, aber auch zur Behandlung von Entzugssyndromen (Alkohol!) und Delirien. Sie sind auch antiepileptisch wirksam [1, 2]. Außerdem werden Benzodiazepine in weiten Bereichen der inneren Medizin oder zur Prämedikation in der Anästhesie und vor diagnostischen oder therapeutischen Eingriffen eingesetzt (z. B. Midazolam). Benzodiazepine sind keine Antidepressiva, werden aber häufig als Komedikation bei Depressionen verordnet. Metaanalytische Studien haben gezeigt, dass diese Kombination nur in der Frühphase der Depressionsbehandlung sinnvoll ist [4]. US-amerikanische Studien zeigten, dass etwa $10 \%$ der Patienten mit Antidepressivabehandlung Benzodiazepine bekommen, wobei sich die Therapieergebnisse nach 6 Monaten nicht unterscheiden [5]. Benzodiazepine sind keine Analgetika, werden aber sehr häufig bei psychosomatischen Störungen, speziell chronischen Schmerzsyndromen eingesetzt, obwohl sie nur beim „burning mouth syndrome“ und beim „stiff person syndrome“ effizient sind [6]. Ansonsten sind 
sie bei Schmerzen meist nicht effektiv, sondern beruhigen nur.

\section{Pharmakologie und Nebenwirkungen}

An diese Stelle soll nur kurz zu den pharmakologischen Grundlagen der BZD Stellung genommen werden [1, 2, 7]. Weltweit sind etwa 35 Präparate im Handel. Sie wirken über inhibitorische GABA-Rezeptoren [8, 9]. Benzodiazepine werden oral aufgenommen, in der Leber metabolisiert und über die Nieren ausgeschieden. Es gibt 2 Stoffwechselwege: Einige Benzodiazepine werden dealkyliert über Cytrochrom P450, demethyliert und hydroxiliert (z. B. Diazepam über Cyp2c19, andere BZD über Cyp3a4, 5 oder 7) und schließlich glucuronidiert, andere Benzodiazepine (Oxazepam, Lorazepam, Lormetazepam, Temazepam, etc.) werden gleich glucuronidiert und über die Nieren ausgeschieden. Die zweite Gruppe hat typischerweise kürzere Halbwertszeiten. Insgesamt haben viele Benzodiazepine pharmakologisch aktive Metabolite oder sind Pro-Drugs. Zu den Anxiolytika zählen Alprazolam, Bromazepam, Lorazepam, Ozazepam, Prazepam oder Tetrazepam, zu den Hypnotika Flurazepam, Flunitrazepam, Midazolam, Nitrazepam, Temazepam [3]. Diazepam wird auch zu den Anxiolytika gezählt [7], wirkt aber auch sehr sedierend.

Ob verschiedene Benzodiazepine ein unterschiedlich hohes Missbrauchspotenzial haben, wird kontrovers diskutiert [10, 11]. Lader et al. [12] postulierten z. B. ein höheres Missbrauchspotenzial von Anxiolytika wie Alprazolam bzw. von kurzwirksamen BZD. Letztlich ist aber nicht belegt, dass kurzwirksame Benzodiazepine ein generell höheres Missbrauchsrisiko haben. Gesichert ist nur das hohe Missbrauchspotenzial des starken Hypnotikums Flunitrazepam, das in vielen Ländern deswegen besonders reguliert bzw. unter das Betäubungsmittelgesetz gestellt wurde [1, 10, 13]. Auch Z-Drugs wirken über den gabaergen Rezeptor, haben aber eine etwas andere Pharmakologie [13]. Zolpidem ist als Prototyp der Z-Drugs ein Agonist am GABA-A-Rezeptor mit hoher Affinität zur $\alpha 1-U n-$ tereinheit des GABA-A-Benzodiazepinrezeptors [14-16]. Zaleplon [17] ist mittlerweile vom Markt genommen. Zopiclon und Eszoplicon werden ebenfalls zur Behandlung der Insomnie eingesetzt [18]. Zahlreiche Therapierichtlinien empfehlen Benzodiazepine genauso wie Z-Drugs nur zur Kurzzeitbehandlung der Insomnie [1, 2].

Neurobiologisch ist gesichert, dass Benzodiazepine, wie auch andere Drogen, indirekt über GABA-Rezeptoren zu einer Dopaminausschüttung im mesolimbischen Belohnungssystem führen, das für Suchtentwicklungen entscheidend ist [19]. Zuletzt wurde die Bedeutung von Sisha7 für die BZD-Wirkung diskutiert [20]. GABA-Rezeptoren, die im limbischen System lokalisiert sind, stellen die mit Abstand am weitesten verbreiteten inhibitorischen Rezeptoren im ZNS dar und ihre Aktivierung führt indi- rekt zu einer Dopaminausschüttung im mesolimbischen Bereich [21]. Durch eine Reihe von eleganten Experimenten konnte gezeigt werden, dass die $\alpha 1$-Untereinheit des GABA-A-Rezeptors für die Entwicklung einer physiologischen Abhängigkeit von Benzodiazepinen eine entscheidende Rolle spielt.

Benzodiazepine sind sehr sichere Substanzen und haben eine ausgesprochen geringe Organtoxizität. Tödliche Monointoxikationen sind sehr selten, auch tödlich verlaufende Entzüge. Problematisch ist für viele Medikamente, speziell Hypnotika, ein Hangover am nächsten Morgen (nach deutlicher Sedierung), außerdem eine oft zu starke Sedierung, die gerade bei Älteren mit einem Risiko von Stürzen und Unfällen einhergeht. Auch die Fahrtauglichkeit ist beeinträchtigt, speziell bei BZD-Hypnotika, obwohl sich die neurokognitiven Defizite bei langer Einnahme abschwächen bzw. der Patient möglicherweise an die Wirkungen der Medikamente habituiert [22]. Ob Benzodiazepine auch einen kognitiven Abbau induzieren können oder das Risiko für Demenzen inklusive der Alzheimererkrankung erhöhen, wird kontrovers diskutiert, allerdings gibt es einige Befunde in diese Richtung [23-25]. Generell ist die Einnahme von ZNS „depressants“ bei Älteren mit schlechteren kognitiven Leistungen assoziiert.

\section{Medikamentenabhängigkeit}

Im Prinzip gelten für schädlichen Gebrauch oder Missbrauch und Abhängigkeit von verschreibungspflichtigen Medikamenten wie Benzodiazepine und Hypnotika dieselben diagnostischen Grundlagen wie für die Abhängigkeit von Alkohol oder illegalen Drogen. In der ICD-10 sowie der in Kürze eingeführten ICD-11 wird schädlicher Gebrauch und Abhängigkeit von Sedativa und Hypnotika unterschieden [1, 2], während das DSM-5 [26] die kategoriale Unterscheidung Missbrauch und Abhängigkeit zugunsten eines dimensionalen Konzeptes aufgegeben hat. Für die Diagnose einer Substanzstörung werden 11 Kernsymptome definiert. Sind 2 oder 3 der diagnostischen Symptome erfüllt kann man von einer milden, bei 4 bis 5 von einer moderaten, bei 6 oder mehr von einer schweren Substanzgebrauchsstörung ausgehen ( $\triangleright$ Kasten „DSM-5-Symptome“). Sowohl ICD-11 als auch DSM-5 postulieren für legale wir illegale Süchte dieselben diagnostischen Kriterien. Während Toleranzentwicklung, Kontrollverlust oder berauschende, psychotrope Effekte davon nicht berührt werden, spielt dies für die als diagnostische Symptome genannten psychosozialen Folgeschäden und Verhaltensmerkmale eine große Rolle. Insofern ist diskutabel, ob die gleichen diagnostischen Kriterien wie z. B. für Heroin- oder Amphetaminmissbrauch für legale, verschreibungspflichtige Medikamente sinnvoll sind. Aus einer BZD-Sucht resultieren häufig andere Verhaltensmerkmale als aus anderen Suchtformen ( $\triangleright$ Kasten „Klinische Hinweise“). 


\section{DSM-5-SYMPTOME}

Störungen im Zusammenhang mit Sedativa, Hypnotika oder Anxiolytika

- Konsum in größeren Mengen und länger als beabsichtigt

$\mathrm{P}$

- Anhaltender Wunsch oder erfolge Versuche Konsum zu reduzieren oder zu kontrollieren (Kontrollverlust) $P$

- Hoher Zeitaufwand für Beschaffung, Konsum P, S

- Craving, starkes Verlangen P, B

- Konsum trotz Versagen bei Erfüllung wichtiger Verpflichtungen $S$

- Konsum trotz sozialer oder zwischenmenschlicher Probleme $\mathrm{S}$

- Soziale, berufliche, Freizeitaktivitäten werden vernachlässigt $S$

- Konsum trotz körperlicher Gefährdung (z. B. Autofahren) S

- Konsum trotz körperlicher, psychischer Probleme $\mathrm{B}, \mathrm{P}$

- Toleranzentwicklung

B

- Entzugssymptome

B

Erklärung

Ursache oder Schädigung auf folgender Ebene: B: biologisch, physiologisch, P: psychisch, S: sozial

\section{KLINISCHE HINWEISE}

Missbrauch oder Abhängigkeit von Benzodiazepinen oder Z-Drugs

- Tendenz zur Dosiserhöhung, Toleranzentwicklung

- Mangelnde Compliance, Drängen auf Verschreiben bestimmter Medikamente

- Besuche von Apotheken oder Notfallambulanzen unter Angabe „verlorenes Rezept“

- Abwehr von Änderungen der Medikation, auch bei fehlender Besserung

- Horten von Medikamenten

- Rezeptfälschungen, Stehlen oder Borgen von Medikamenten

- Doktor-Hopping (oder -Shopping), mehrere Ärzte werden um Rezepte gebeten

- ursprüngliche Indikation verliert an Bedeutung

- Konzentrationsstörungen, Wesensänderungen

- Zunahme von Angst, Depression, Schlafstörungen, Alpträume

- Einnahme anderer Suchtstoffe, psychotroper Substanzen

- Ggf. i.v. Missbrauch

\section{Klinische Symptome von Missbrauch und Abhängigkeit}

In der S3-Leitlinie für „Medikamentenbezogene Störungen“ (gemeint Missbrauch und Abhängigkeit von verschreibungspflichtigen Medikamenten) sind eine Reihe von spezifischen und unspezifischen Symptomen aufgelistet, die jenseits der ICD-10/-11 - und DSM-5-Kriterien für eine Abhängigkeitsentwicklung von Medikamenten sprechen ( $\triangleright$ Kasten „Entzugssymptome“). Typische psychische und Verhaltensmerkmale von BZD-Missbrauch sind von Autoren wie Ashton [27-29] dargestellt worden. Typisch ist vor allem das Horten von Medikamenten, Doctor-Shopping (oder Hopping), Rezeptsammlungen und Einreichen von Rezepten bei verschiedenen, auch Versandapotheken, das wiederholte Aufsuchen von Ambulanzen oder fremden Ärzten, um Medikamente einzufordern oder verlorene Rezepte zu beklagen und Angst vor jeder Medikamentenreduktion.

\author{
ENTZUGSSYMPTOME \\ Sedativa, Hypnotika und Anxiolytika \\ Psychisch \\ - Angst \\ - Schlafstörungen \\ - Innere Unruhe, Anspannung \\ - Depression \\ - Erhöhte Irritabilität \\ - Kognitive Störungen \\ - Merkfähigkeits- und Konzentrationsstörungen \\ - Schwerer Verlauf \\ - Psychose \\ - Verwirrtheitssyndrom \\ - Delir \\ Vegetativ \\ - Zittern \\ - Übelkeit, Erbrechen \\ - Schwitzen \\ - Motorische Unruhe \\ - Erhöhter Puls, Blutdruck \\ - Dyspnoe \\ - Kopfschmerzen \\ - Muskelzuckungen oder -verspannungen \\ Neurologisch, somatisch \\ - Epileptische Anfälle \\ - Wahrnehmungs- und Perzeptionsstörungen \\ - Hyperakusis \\ - Photophobie \\ - Dysästhesien \\ - Störungen der Motorik
}

\section{Prävention}

In den meisten Therapierichtlinien wird höchstens eine mehrwöchige Medikation von Benzodiazepinen oder 
Z-Drugs empfohlen [1, 7]. Mehrmonatige Behandlungen werden wegen des Risikos von Toleranzentwicklungen, Dosissteigerungen und physischer und psychischer Abhängigkeit sehr kritisch gesehen [11, 12, 29-31].

Zur Anwendung von BZDs gilt die 5-K-Regel:

- klare Indikation,

- kleinstmögliche Dosis,

- kürzester möglicher Zeitraum,

- kein abruptes Absetzen,

- Kontraindikationen sind zu beachten (siehe S3-Leitlinie).

Natürlich führen auch Veränderungen der Verfügbarkeit und Zulassungsregeln zu einem veränderten Verschreibungsverhalten, auch wenn man diese nicht überschätzen sollte, wie z. B. Erfahrungen aus Irland gezeigt haben [32].

\section{Epidemiologie}

Die Datenbasis über Missbrauch und Abhängigkeit von verschreibungspflichtigen Medikamenten ist sehr viel schlechter als die von Alkohol oder Drogen. Oft wird aus der Verschreibung oder Langzeitanwendung von Benzodiazepinen auf die Zahl der Abhängigen geschlossen, was methodisch fragwürdig ist. Häufig wird für Deutschland eine Zahl von 1 bis 1,2 Mio. Menschen mit Missbrauch oder Abhängigkeit von Sedativa und Hypnotika angegeben [1-3, 33, 34]. International ist die Datenbasis besser. Von 1999 bis 2014 war der Konsum von BZD- und Non-BZD-Hypnotika in den USA deutlich gestiegen, vor allem wegen häufigerer Langzeitverschreibungen [35]. In den USA nehmen 12,5\% der Erwachsenen pro Jahr Benzodiazepine ein, 2,1\% missbrauchen sie. Von den BZD-Konsumenten missbrauchen diese $17,1 \%, 1,5 \%$ haben eine BZD-Konsumstörung (benzodiazepine use disorder). Der Missbrauch geht mit anderem Substanzkonsum, Suizidalität, psychischen Störungen und Notfalleinweisungen einher. In England hatten in einem Quartal 3,1 \% der Bevölkerung ein Rezept für ein Benzodiazepin, 2,3 \% für ein Z-Drug [36]. Tatsache ist, dass die Verschreibung von Benzodiazepinen zu Lasten der Krankenkassen in den letzten Jahren deutlich gefallen ist, allerdings bei einem gleichzeitigen Anstieg der Rezeptierungen auf Privatrezepten und eines Anstiegs von Verschreibungen der Z-Drugs [37]. Derselbe Trend ist, mit einigen Ausnahmen, auch international erkennbar [35, 38-40], sehr schön gezeigt z. B. für Finnland [41]. Eine Ausnahme ist Kolumbien [42]. Ergebnisse aus Japan zeigen, dass $10 \%$ der Patienten mit Erstverschreibungen von Hypnotika auch nach 12 Monaten die Substanz noch einnehmen [43].

Besonders häufig ist ein BZD-Langzeitkonsum bei Patienten mit chronischen Schmerzerkrankungen z. B. Kopfschmerzen. Daten aus Frankreich zeigen, dass etwa $30 \%$ der Hypnotika-Verschreibungen und $20 \%$ der Anxiolytika nicht leitliniengerecht sind [44]. Viele dieser Verschrei- bungen erfolgen nicht durch Psychiater [45-47]. Daten der National Survey on Drug Use and Health zeigten, dass 2,1 \% der Erwachsenen Benzodiazepine missbraucht haben, fanden aber nur eine Prävalenz von BZD-Konsumsubstanzstörungen von 0,2\% [46]. Ein systematisches Review ergab allerdings für die USA eine Missbrauchs- und Abhängigkeitsrate von Benzodiazepinen und Tranquilizern von 2,2\% [48]. Weltweit wurden ebenfalls Raten von $2,2 \%$ gefunden [49]. In vielen Studien fand sich eine Assoziation vom niedrigen sozioökonomischen Status und BZD-Langzeitbehandlungen [48]. Es ist etwas umstritten, ob das Missbrauchspotenzial von Z-Drugs kleiner ist als das von Benzodiazepinen [17, 18, 50-52]. Es ist aber sicher ebenfalls beträchtlich und ausgesprochene Hochdosisabhängigkeiten werden auch hier beobachtet [5355]. Auch für Z-Drugs gilt ein Risiko für kognitive Beeinträchtigungen und Unfälle [56, 57].

\section{Risikofaktoren und sozioökonomische Korrelate des Langzeitkonsums}

In vielen Untersuchungen wurde gezeigt, dass die Rate der BZD-Verschreibungen bei Frauen deutlich höher ist als bei Männern, und das Risiko für Langzeitverschreibungen steigt mit dem Alter exponentiell an [58, 59]. In einer niederländischen Studie zu Angst und Depression war BZD-Abhängigkeit mit Schlafstörungen, antidepressiver Behandlung und Alkoholabhängigkeit assoziiert [60]. Patienten mit anderen Suchterkrankungen, z. B. Alkoholismus oder Polytoxikomane sind Risikopatienten für BZD-Missbrauch [61]. Besonders betroffen sind Opiatabhängige [62-69], wobei Raten von etwa 21-66\% berichtet wurden [66-69]. Benzodiazepine spielen auch für die Mortalität bei Opioiden (overdose death) eine erhebliche Rolle [70-72]. Auch die Retentionsrate ist bei Patienten in der Substitutionsbehandlung bei BZD-Konsum kleiner [73]. Alkohol und Benzodiazepine sind erhebliche Risikofaktoren für tödliche Opiatintoxikationen [74]. Besonders bedenklich ist das Risiko bei Älteren (vor allem Frauen) mit Benzodiazepinen langfristig behandelt zu werden [58, 71, 75, 76]. Über BZD-Langzeitbehandlungen bei Kindern und Jugendlichen ist dagegen weniger bekannt [77]. Die epidemiologischen Daten belegen eine Reihe von funktionellen Konsequenzen von BZD-Missbrauch oder -abhängigkeit. Dazu gehören Suizidalität, Risiko für Infektionskrankheiten, Kriminalität, geringe Lebensqualität und schlechte Prognose bei der Behandlung von Suchterkrankungen [49].

\section{Klinisches Bild der}

\section{Benzodiazepinabhängigkeit}

Entzugserscheinungen und Abhängigkeit von Benzodiazepinen wurden früh beschrieben [78], aber lange ignoriert. Toleranzentwicklungen und Dosissteigerungen sind sehr häufig, aber anders als bei anderen Rauschdrogen kann es bei Benzodiazepinen zu einer Niederdosis- 
abhängigkeit (low dose dependence), also einer psychischen und physischen Abhängigkeit von Benzodiazepinen ohne Dosissteigerung und Toleranzsteigerung, kommen. In den meisten Fällen wird bei Langzeitkonsum von Benzodiazepinen aber die Dosis deutlich ansteigen und die Ursprungsindikation verliert ihre Bedeutung. So werden z. B. Hypnotika zunehmend über den Tag genommen, um überhaupt funktionsfähig zu bleiben oder Entzugssymptome zu vermindern. Das BZD-Entzugssyndrom ist ausgesprochen vielgestaltig und entspricht im Prinzip dem von Alkohol oder anderen Hypnotika. Es ist letztlich auf eine verminderte Funktion (Down-Regulation) des GABAergen Systems und einer vermehrten Freisetzung exzitatorischer Neurotransmitter zurückzuführen. Pathognomonische Befunde fehlen, aber es gibt eine Reihe von typischen Symptomen, z. B. die Überempfindlichkeit für Licht, Geräusche und Berührung oder andere Wahrnehmungs- und Perzeptionsstörungen. Beim plötzlichen Absetzen kann es häufig zu epileptischen Anfällen kommen, seltener sind Psychosen und Delire, vor allem bei Hochdosisabhängigkeit. Unruhe, Angst, Stimmungsschwankungen oder Depressionen sind dagegen häufig, Schlafstörungen mehr oder weniger obligat. Auch für Non-BZD-Hypnotika wie Eszopiclon sind Entzugserscheinungen nach Absetzen beschrieben worden [79]. Toxikologische Urinkontrollen können bei der Diagnose helfen und den Missbrauch anderer Substanzen ausschließen. Die Bestimmung von Plasmakonzentration bei BZD-Abhängigkeit ist nicht üblich, Haaranalysen nur bei forensisch relevanten Fragestellungen wegweisend.

\section{Differenzialdiagnose}

Diese ist sehr breit und umfasst eine Reihe von neurologischen und somatischen Erkrankungen. Dazu gehören:

- Entzug von anderen Substanzen (Alkohol, Drogen),

- Epilepsie,

- Hirntraumata, Enzephalitis,

- Delirien anderer Genese (metabolisch, toxisch),

- affektive Erkrankungen,

- andere psychiatrische Erkrankungen (Angst, bipolare Störung, Schizophrenie),

- diverse somatische Erkrankungen je nach klinischem Erscheinungsbild (z. B. Herzinfarkt bei starker Angst).

\section{Therapie}

Es gibt relativ wenige klinisch kontrollierte Untersuchungen zur Frage der optimalen Behandlung der BZD-Abhängigkeit. Es existieren eine Reihe von kritischen Reviews, Metaanalysen und evidenzbasierten Leitlinien [1, 2, 31, 80-86, aktuelle AWMF-S3-Leitlinie Medikamentenbezogene Störungen], wichtig sind die Cochrane-Analysen [80, 87]. Gesichert ist, dass das abrupte Absetzen von Benzodiazepin nach Langzeitgabe speziell bei hohen Dosen zu plötzlich auftretenden Entzugssymptomen führen kann, die klinisch bedeutsam und für den Patienten kaum zu tolerieren sind. Insbesondere das Risiko von epileptischen
Anfällen und Entzugspsychosen ist erheblich. Deswegen ist das schrittweise Absetzen von Benzodiazepinen (tapering off) der Goldstandard in der Behandlung der BZD-Abhängigkeit. Dabei werden die Medikamente über mehrere Wochen, abhängig von der Initialdosis, schrittweise reduziert. Bei zu raschem Absetzen sind die Entzugssymptome häufig nicht tolerabel und der Patient bricht die Behandlung ab. Ein zu vorsichtiger Entzug ist für den Patienten dagegen oft ein „endloser Horror“. Deswegen ist es in vielen günstiger eine bestimmte Behandlungsdauer (z. B. 6 Wochen) abzusprechen, damit die Entzugsbehandlung nicht der „morbid focus“ des Patienten wird [30]. Viele BZD-Entzugsprotokolle gehen von einer $25 \%$ igen Reduktion alle 1-2 Wochen aus [88], für viele Ärzte und Patienten sicher ein realistischer Behandlungsansatz. Der Wechsel von Benzodiazepinen mit kurzer HWZ zu langer HWZ (Diazepam) wird immer wieder diskutiert und ist empirisch nicht ausreichend belegt [31]. Beim Entzug mehrerer Substanzen ist die Umstellung auf eine langwirkende Substanz sinnvoll.

Es gibt keine Medikamente, die für die Entzugsbehandlung der BZD-Abhängigkeit zugelassen sind. In ihrer Übersicht fanden Welsh et al. [89] nur schwache Hinweise für die Wirksamkeit einzelner Substanzen bei BZD-Hypnotika und Anxiolytikaentzug. Für die Behandlung von Schlafstörungen bieten sich entweder sedierende Trizyklika oder Antihistaminika an, wie auch in der Leitlinie zur Behandlung von Schlafstörungen empfohlen wird [90]. Entscheidend ist eine gute Schlafhygiene [91]: Nicht zu spät ins Bett gehen, nicht zu schwer essen oder Alkohol vor dem Schlafengehen, keine Horrorfilme sehen etc. In der erwähnten Therapierichtlinie zur Behandlung der Hyposomnie werden z. B. Trazodon, Doxepin, Mirtazapin und Trimipramin als mögliche Medikamente genannt [90]. Eine Cochrane-Analyse zur Effizienz von Antidepressiva bei Insomnie über 23 Studien zeigte nur eine begrenzte Wirksamkeit [92]. Ähnliche Befunde werden in anderen Übersichten mitgeteilt [93, 94].

Antidepressiva werden auch sonst in der Behandlung der BZD-Abhängigkeit häufig eingesetzt, durchaus mit gemischten Ergebnissen [95, 96]. Dennoch dürften sie die realistischste Behandlungsoption im BZD-Entzug sein. Weniger gut untersucht und allenfalls Second-line-Medikamente sind Carbamazepin [97] und Gabapentin [66, $98,99]$. Gabapentin ist in diesem Kontext nicht uninteressant, hat aber ein gewisses Missbrauchspotenzial (S3-Leitlinie). Ein weiterer Kandidat ist der GABA-B-Agonist Baclofen, der, bei sehr heterogener Datenbasis, vor allem in Frankreich zur Behandlung der Alkoholabhängigkeit eingesetzt wird [100, 101]. Hier sind Studien angedacht, es liegen aber nur wenige kasuistische Befunde zur Wirksamkeit bei BZD-Abhängigkeit vor [102]. Auch Melatonin oder melatonerge Substanzen haben eine geringe Wirkung gezeigt [103]. Inwieweit Pregabalin eine realistische Behandlungsalternative darstellen wird, muss 
die Zukunft zeigen. Pregabalin wurde im BZD-Entzug in Dosen von 150 bis $600 \mathrm{mg}$ eingesetzt [103]. Die Substanz hat allerdings ein erhebliches Missbrauchspotenzial [104], insbesondere bei Patienten mit anderen Suchterkrankungen [87]. Insgesamt kann Pregabalin noch nicht als Medikation im BZD-Entzug empfohlen werden [105]. Keine große Bedeutung haben Betablocker [106] oder Valporat [107], auch wenn dies gelegentlich empfohlen wird [108]. Experimentell wurde eine „rapid detoxification“ von Benzodiazepinen mit Flumazenil, einem BZD-Antagonisten $[109,110]$ empfohlen. Zuletzt wurde wieder eine Arbeit publiziert, bei der über 7 Tage Flumazenil $1 \mathrm{mg} /$ Tag s.c. zum BZD-Entzug gegeben wurde [111]. Es gibt aber erhebliche medizinische Risiken, z. B. epileptische Anfälle [112]. In Analogie zur Substitutionsbehandlung von Opioiden wurde eine Substitutionsbehandlung bei BZD-Abhängigkeit diskutiert, z. B. mit Clonazepam, speziell bei „High-dose“-Konsumenten [113]. Letztlich liegen hier keine belastbaren Befunde vor.

\section{Psychotherapie}

Die Therapieempfehlungen bei Medikamentenabhängigkeit orientieren sich stark an denen anderer Suchtformen, insbesondere Alkoholismus. Allerdings gibt es Besonderheiten. Der erste Schritt ist immer, dass (Sucht-)Problem zu erkennen und, wenn nötig mehrfach, anzusprechen und mögliche Behandlungsalternativen vorzuschlagen $[28,113]$. Relativ gut belegt ist die Effizienz von „brief interventions “ bei Suchterkrankungen [114] und BZD-Missbrauch. Sie werden vor allem beim Hausarzt oder in (Notfall-)Ambulanzen eingesetzt, aber auch im Krankenhaus, und reichen vom persönlichen Ratschlag (advice) über schriftliches Aufklärungsmaterial, Selbsthilfebroschüren und Material über die Folgen und Risiken des Konsums bis hin zur Vermittlung von Beratungsstellen oder Therapeuten. Möglicherweise haben Patienten, die die BZD-Rezepte vom Hausarzt bekommen, eine bessere Prognose als die, die ihre Rezepte von anderen Ärzten bekommen [115]. Die Effizienz solcher Interventionen im Primary-care-Bereich ist gut belegt $[116,117]$, wie zuletzt eine Metaanalyse zeigte, die 8 Studien mit 2071 Patienten umfasste [117]. Teilnehmer in einer Interventionsgruppe wiesen nach 6 Monaten 2,73-mal häufiger eine BZD-Abstinenz auf, es gibt allerdings auch negative Studien [118]. Eine „personalisierte“ Herangehensweise wird generell bevorzugt, mit Aufklärung über die gesundheitlichen und sozialen Folgen des Konsums und einem schrittweisen BZD-Absetzen [119].

\section{Psychosoziale Therapien und Psychoedukation}

Eine spezifische Psychotherapie für Abhängigkeit von Hypnotika und Tranquilizern gibt es nicht [1, 2]. Wichtig sind Setting-Effekte: Anders als bei anderen Süchten werden die meisten Patienten nicht im eigentlichen Suchthilfesystem, sondern eher beim Hausarzt, Psychiater oder in der Psychosomatik behandelt [1-3]. Die Anzahl kontrollierter Studien in diesem Bereich ist überschaubar [107,
120, 121]. Generell wirkt Psychotherapie bei Patienten mit BZD-Abhängigkeit und -entzug besser als „treatment as usual“ [122, 123].

Zur Psychoedukation liegen einige Befunde vor [119]. Gerade bei Medikamentenabhängigkeit kommt der Vermittlung von Wissen über die Grunderkrankung, Behandlungsmöglichkeiten und Wirkungen von Medikamenten eine große Rolle zu. Welche Rolle spielt das Medikament im Leben, gibt es Behandlungsalternativen [124]? Ein systematischer Review zeigte eine Wirksamkeit psychoedukativer Maßnahmen, z. T. in Kombination mit kognitiver Verhaltenstherapie [125].

\section{Motivationale Therapien und KVT}

Basierend auf dem transtheoretischen Model von Prochaska und DiClemente [126] wurde das „motivational interviewing“ entwickelt [127], das verschiedene Phasen der Willensbildung und Veränderungsbereitschaft beschreibt. Man kann dies auch auf die Therapie von Medikamentenabhängigen übertragen, aber es sind nur wenige Befunde verfügbar [115].

KVT ist bei Suchterkrankungen intensiv untersucht worden [128]. Bei Medikamentenabhängigkeit liegt der Fokus z. B. auf der Analyse der Funktion des eingenommenen Medikaments, Umgang mit Stress, negativen Gefühlen oder psychosomatischen Beschwerden [124]. KVT kann als Einzel- oder Gruppentherapie angeboten werden. Der Patient lernt das „Nein-Sagen“. Wichtig sind Selbstwirksamkeitsstrategien, der Glaube und das Vertrauen, mit bestimmten Beschwerden auch ohne eine Tablette fertig zu werden. Erkennen, Vermeiden und Umgang mit Risikosituationen gehört dazu [1-3, 119]. Morgan et al. [129] konnten in einer randomisierten kontrollierten Studie die Wirksamkeit von KVT bei Patienten mit Schlafstörungen zeigen $(n=209)$. Die Kombination von KVT mit motivationalen Therapien wird bei BZD-Abhängigkeit empfohlen [83], oft auch in Kombination mit Entspannungsverfahren wie progressive Muskelrelaxation oder autogenem Training. Andere Therapien richten sich nach der zugrunde liegenden Erkrankung, z. B. einer Angststörung.

\section{Ergebnisse der Therapieforschung}

Eine Cochrane-Analyse [80] untersuchte die Effizienz des „motivational enhancement“ [127] gegenüber Standardtherapie und KVT während der BZD-Reduktion. Insgesamt waren, bei überschaubarer Datenbasis, die Befunde für KVT am besten. Für komorbid psychisch erkrankte Menschen gibt es wenig Befunde. KVT wird empfohlen $[30,130]$. Eine Metaanalyse zeigte eine generelle Wirksamkeit von Psychotherapie bei Älteren mit BZD-Entzug [125]. Psychoedukation plus Psychotherapie hat sich als wirksam erwiesen $[125,131]$. Die Prognose bei BZD-Langzeitkonsum ist nicht schlecht. Untersuchun- 
gen von Rickels et al. [132] zeigten 73\% Abstinenz nach 2-5 Jahren bei Patienten, die ein Therapieprogramm mit schrittweisem Absetzen von BZD erfolgreich beendet hatten. Andere Nachuntersuchungen zeigten weniger günstige Ergebnisse [133], während die in diesem Bereich sehr erfahrene Autorin Ashton und Kollegen [27] eine 85 \%ige Abstinenz in einer Stichprobe von 50 Patienten fand. Insgesamt liegen nur wenige kontrollierte randomisierte Therapiestudien vor [84, 130,134-136]. De Gier et al. [120] legten eine große Studie vor und fanden nach „minimal intervention“ eine Abstinenzrate von 58,8\%. Einige Untersuchungen haben sich der Frage der Therapie Älterer mit BZD-Abhängigkeit gewidmet [128]. Es liegt eine Monografie zu dem Thema vor [137]. Sie sind durch Verletzungen, Stürze und kognitiven Abbau besonders gefährdet, gleichzeitig besonders häufig betroffen. Genaue Aufklärung bzw. Psychoedukation, KVT und ggf. das Angebot pharmakologischer Alternativen (z. B. bei Insomnie) werden empfohlen [125, 138]. Da ein Risiko z. B. für Delire existiert [139], wird zu einem eher behutsamen Abbau geraten.

\section{FAZIT}

Benzodiazepine und Z-Drugs sind ausgesprochen effektive und sichere Medikamente [140, 141]. Hauptproblem und Risiko ist die erhebliche Toleranz und Gewöhnung bei längerer Anwendung, weswegen der Prävention (genaue Indikationsstellung, kurze Behandlungsdauer) eine entscheidende Bedeutung zukommt. Risikogruppen für Suchtentwicklungen sind Patienten mit anderen Süchten, schweren psychischen und psychosomatischen Störungen, Schmerz-Patienten und medizinisches Personal. Frauen und ältere Menschen sind viel häufiger betroffen.

Die Bedeutung von „brief interventions“ bei BZD-Abhängigkeit ist gesichert. Ein schrittweises Absetzen bei Langzeitkonsum ist in jedem Fall sinnvoll. Einen Goldstandard in der begleitenden Psychopharmakotherapie gibt es nicht, oft werden Antidepressiva eingesetzt. Aufklärung und Psychoedukation, (kognitive) Verhaltenstherapie und motivationale Ansätze spielen klinisch eine wichtige Rolle. Die übrige Therapie richtet sich nach der (psychischen) Grunderkrankung. Die wenigen Langzeituntersuchungen rechtfertigen einen gewissen Optimismus hinsichtlich der langfristigen Abstinenz.
Interessenkonflikt

\section{Erklärung zu finanziellen Interessen}

Forschungsförderung erhalten: nein; Honorar/geldwerten Vorteil für Referententätigkeit erhalten: nein; Bezahlter Berater/interner Schulungsreferent/Gehaltsempfänger: nein; Patent/Geschäftsanteile/Aktien (Autor/Partner, Ehepartner, Kinder) an Firma (Nicht-Sponsor der Veranstaltung): nein; Patent/Geschäftsanteile/Aktien (Autor/Partner, Ehepartner, Kinder) an Firma (Sponsor der Veranstaltung): nein.

Erklärung zu nicht finanziellen Interessen

Der Autor gibt an, dass kein Interessenkonflikt besteht.

\section{Korrespondenzadresse}

Prof. Dr. med. Michael Soyka

Psychiatrische Klinik München, Universität München Nußbaumstr. 7, 80336 München, Deutschland michael.soyka@med.uni-muenchen.de

Literatur

[1] Soyka M. Medikamentenabhängigkeit. Stuttgart: Schattauer; 2015

[2] Soyka M, et al. (Hrsg.). Suchtmedizin. München: Elsevier; 2019

[3] Soyka M, Batra A. Benzodiazepin-Abhängigkeit. In: Vorderholzer U, Hohagen F (Hrsg.) Therapie psychischer Erkrankungen State of the Art. München: Urban und Fischer; 2021

[4] Ogawa Y, Takeshima N, Hayasaka Y, et al. Antidepressants Plus Benzodiazepines for Adults with Major Depression. Cochrane Database Syst Rev 2019; 6: CD001026

[5] Bushnell GA, Stürmer T, Gaynes BN, et al. Simultaneous Antidepressant and Benzodiazepine New Use and Subsequent Long-Term Benzodiazepine Use in Adults With Depression, United States, 2001-2014. JAMA Psychiatry 2017; 74: 747-755

[6] Wright SL. Limited Utility for Benzodiazepines in Chronic Pain Management. A Narrative Review. Adv Ther 2020; 37: 2604-2619

[7] Benkert O, Hippius H. Kompendium der Psychiatrischen Pharmakotherapie. Berlin: Springer; 2017

[8] Altamura CA, Moliterno D, Paletta S, et al. Understanding the pharmacokinetics of anxiolytic drugs. Expert Opin Drug Metabol Toxicol 2013; 9: 423-440

[9] Castellano DD, Shephard R, Lei W. Looking for Novelty in an „Old“ Receptor: Recent Advances Toward Our understanding of GABAARs and their Implications in Receptor Pharmacology. Front Neurosci 2021; 14: doi:10.3389/ fnins.2020.616298

[10] Cloos JM, Bocquet V, Portal-Rolland I, et al. Hypnotics and Triazolobenzodiazepinse - Best Predictors of High-Dose Benzodiazepine Use: Results from the Luxembourg national Health Insurance Registry. Psychother Psychosom 2015; 84; 273-280

[11] Ait-Daoud N, Hamby AS, Sharma S, et al. Review of Alprazolam Use, Misuse, and Withdrawal. J Addict Med 2018; 12: 4-10 
[12] Lader M, Tylee A, Donoghue J. Withdrawing benzodiazepines in primary care. CNS Drugs 2009; 23: 19-34

[13] Atkin T, Comai S, Gobbi, G. Drugs for Insomnia Beyond Benzodiazepines: Pharmacology, Clinical Applications, and Discovery. Pharmacol Rev 2018; 70: 197-245

[14] Faccini M, Tamburin S, Casari R, et al. High-dose lormetazepam dependence: strange case of Dr. Jekyll and Mr Hyde. Intern Emerg Med 2019; 14: 1271-1278

[15] Greenblatt D, Roth T. Zolpidem for Insomnia. Expert Opin Pharmacother 2012; 13: 879-893

[16] Liappas I, Malitas P, Dimopoulos N, et al. Zolpidem Dependence Case Series: Possible Neurobiological Mechanisms and Clinical Management. J Psychopharmacol 2013; 17: 131-135

[17] Dooley M, Plosker G. Zaleplon: A Review of Its Use in the Treatment of Insomnia. Drugs 2000; 60: 413-445

[18] Rösner S, Englbrecht C, Wehrle R, et al. Eszopiclone for Insomnia. Cochrane Database Syst Rev 2018; 10: CD010703

[19] Luscher C, Ungless, M. The mechanistic classification of addictive drugs. PLoS Med 2006; 3: e437

[20] Han W, Li J, Pelkey K, et al. Shisha7 is a GaBaA receptor auxillary subunit controlling benzodiazepine actions. Science 2006; 366: 246-250

[21] Tan K, Brown M, Labouebe G, et al. Neural bases for addictive properties of benzodiazepines. Nature 2010; 463: 769-74

[22] Van der Sluiszen N, Vermeeren A, Verster JC, et al. Driving performance of benzodiazepine anxiolytics and hypnotics, Hum Psychopharmacol Clin Exp 2019; 34: e2715

[23] Crowe S, Stranks E. The Residual Medium and Long-term Cognitive Effects of Benzodiazepine Use: An Updated Meta-Analysis. Arch Clin Neuropsychol 2018; 33: 901-911

[24] de Gage S, Moride Y, Ducruet T, et al. Benzodiazepine Use and Risk of Alzheimer's Disease: Case-Control Study. BM] 2014; 5205

[25] Biétry F, Pfeil A, Reich O, et al. Benzodiazepine Use and Risk of Developing Alzheimer's Disease: A Case-Control Study Based on Swiss Claims Data. CNS Drugs 2017; 31: 245-251

[26] American Psychiatric Association. Diagnostic and Statistical manual of Mental Disorders (DSM-5). Wahington: APA; 2013

[27] Ashton $\mathrm{H}$. Benzodiazepine withdrawal: outcome in 50 patients. Br J Addict 1987; 82: 665-671

[28] Ashton $\mathrm{H}$. The Treatment of Benzodiazepine Dependence. Addiction 1994; 89: 1535-1541

[29] Ashton H. Benzodiazepine Abuse. In: Caan W, de Belleroche J (eds.) Drink, Drugs and Dependence From science to clinical practice. New York: Routledge; 2002

[30] Lader M. Benzodiazepines revisited - will we ever learn? Addiction 2011; 106: 2086-109

[31] Soyka M. Treatment of benzodiazepine dependence. N Engl J Med 2017; 376: 1147-1157

[32] Cadogan CA, Bradley CP, Bennett K. Impact of Changes in controlled drugs legislation on benzodiazepine receptor agonist prescribing in Ireland: a repeated cross-sectional study. Eur J Clin Pharmacol 2021. doi.org/10.1007/s00228020-03063-z

[33] Pabst A, Kraus L, Gomes de Matos E, et al. Substanzkonsum und substanzbezogene Störungen in Deutschland im Jahr 2012. Sucht 2013; 59: 321-31

[34] Hoffmann F, Glaeske G. Benzodiazepine Hypnotics, Zolpidem and Zopiclone on Private Prescriptions: Use
Between 1993 and 2012 (in German). Nervenarzt 2014; 85: 1402-1409

[35] Kaufmann CN, Spira AP, Depp CA, et al. Long-Term use of Benzodiazepines and Nonbenzodiazepine Hypnotics 1999-2014. Psychiatr Serv 2018; 69: 235-238

[36] Mardsen J, White M, Annand F, et al. Medicines Associated With Dependence or Withdrawal: A Mixed-Methods Public Health Review and National Database Study in England. Lancet Psychiatry 2019; 6: 935-950

[37] Verthein U, Buth S, Holzbach R, et al. Benzodiazepines and Z-Drugs - Analyses of Ambulatory Prescriptions From 2006 to 2015 (in German). Psychiat Prax 2019; 46: 399-405

[38] Begum M, Gonzalez CD, Bernardo C, et al. Trends in the prescription of drugs used for insomia in Australian general practice, 2011-2018. Br J Gen Practice 2021. doi. org/10.3399/BJGP.2021.0054

[39] Bénard-Laribière A, Noize P, Pambrun E, et al. Trends in incident use of benzodiazepine and Z-drugs in France from 2006 to 2012: a population-based study. Pharmacoepidemiol Drug Safety 2017; 26: 162-169

[40] Holm E, Fosbol E, Pedersen $\mathrm{H}$, et al. Benzodiazepine use in Denmark 1997-2008. Eur Geriatr Med 2012; 3: 299-303

[41] Kurko T, Saastamoinen LK, Tuulio-Henriksson A, et al. Trends in the long-term use of benzodiazepine anxiolytics and hypnotics: A national register study for 2006 to 2014. Pharmacoepidemiol Drug Saf 2018; 27: 674-682

[42] Moreno-Gutierrez P, Gaviria-Mendoza, A, Ochoa-Orozco $S$, et al. Long-term users of benzodiazepines in Colombia. Patterns of use and cessation of treatment. Drug Alcohol Depend 2020; 210: 107962

[43] Enomoto M, Kitamura S, Tachimori $\mathrm{H}$, et al. Long-term use of Hypnotics: Analysis of trends and risk Factors. Gen Hosp Psychiatry 2020; 62: 49-55

[44] Panes A, Pariente A, Bénard-Laribière A, et al. Use of benzodiazepines and z-drugs not compliant with guidelines and associated factors: a population-based study. Eur Arch Psychiatry Clin Neurosci 2020; 271: 3-10

[45] Agarwal SD, Landon B. Patterns in Outpatient Benzodiazepine Prescribing in the United States. JAMA Network Open 2019; 2: E187399

[46] Blanco C, Han B, Jones C, et al. Prevalence and Correlates of Benzodiazepine Use, Misuse, and Use Disorders Among Adults in the United States. J Clin Psychiatry 2018; 79 (6)

[47] Lopéz-Papelayo H, Coma A, Gual A, et al. Call for action: Benzodiazepine prescription Prevalence Analysis Shows Off-label Prescription in one of eleven Citizens. Eur Addict Res 2019; 25: 320-329

[48] Soyombo S, Stanbrook R, Aujla H, et al. Socioeconomic Status and benzodiazepine and z-drug prescribing: a cross-sectional study of practice-level data in England. Fam Pract 2019; 37: 194-199

[49] Votaw V, Geyer R, Rieselbach M, et al. The Epidemiology of Benzodiazepine Misuse: A Systematic Review. Drug Alcohol Depend 2019; 200: 95-114

[50] Curreen M, Lidmilla J. Zopiclone: Is there Cause for Concern in Addiction Services and General Practice? Int J Risk Saf Med 2014; 26: 183-189

[51] Soyka M, Bottlender M, Möller HJ. Epidemiological Evidence for a Low Abuse Potential of Zolpidem. Pharmacopsychiatry 2002; 33: 138-141

[52] Hajak G, Müller W, Wittchen H, et al. Abuse and Potential for the Non-Benzodiazepine Hypnotics Zolpidem and 
Zopiclone: A Review of Case Reports and Epidemiological Data. Adiction 2003; 98: 1371-1378

[53] Lugoboni F, Mirijello A, Faccini M, et al. Quality of life in a cohort of high-dose benzodiazepine dependent patients. Drug Alcohol Depend 2014; 142: 105-109

[54] Gunja N. The clinical and forensic toxicology of Z-Drugs. Med Toxicol 2013; 9: 155-162

[55] Victorri-Vigneau C, Géradin M, Rousselet M, et al. Update on Zolpidem Abuse and Dependence. J Addict Dis 2014; 33: $15-23$

[56] Gunja N. In The Zzz zone: the effects of Z-Drugs on human performance and driving. J Med Toxicol 2013; 9: 163-171

[57] Mets MA, de Vries JM, de Senerpont Domis L, et al. Nextday effects of ramelton $(8 \mathrm{mg})$, zopiclone $(7.5 \mathrm{mg})$, and placebo on highway driving performance, memory functioning, psychomotor performance, and mood in healthy adult subjects. Sleep 2011; 34: 1327-1334

[58] Johnson C, Frei C, Downes N, et al. Benzodiazepine and Z-Hypnotic Prescribing for Older People in Primary Care: A Cross-Sectional Population-Based Study. Br J Gen Pract 2016; 66: e410-415

[59] Tevik K, Selbaek G, Engedal K, et al. Factors associated with alcohol consumption and prescribed drugs with addiction potential among older women and men - the Nord-Trondelag health study (HUNT 2 and HUNT 3), a population based study. BMC Geriatr 2019; 19: 113

[60] Manthey I, Lohbeck M, Giltay E], et al. Correlates of benzodiazepine dependence in the Netherlands Study of Depression and anxiety. Addiction 2012; 107: 2173-2182

[61] McHugh R, Geyer R, Karakula S, et al. Nonmedical benzodiazepine use in adults with alcohol use disorder. The role of anxiety sensitivity and polysubstance use. Am J Addict 2018; 27: 485-490

[62] Morel A, Grall-Bronnec M, Bulteau S, et al. Benzodiazepine Dependence in Subjects with Alcohol Use Disorders: What Prevalence? Expert Opin Drug Saf 2016; 15: 1313-1319

[63] Bachhuber M, Hennessy S, Cunningham C, et al. Increasing Benzodiazepine Prescriptions and Overdose Mortality in the United States, 1996-2013. Am J Public Health 2016; 106: 686-8

[64] Backmund M, Meyer K, Henkel C, et al. Co-Consumption of Benzodiazepines in Heroin Users, Methadone-Substituted and Codeine-Substituted Patients. J Addict Dis 2005; 24: $17-29$

[65] Brands B, Blake J, Marsh D, et al. The impact of benzodiazepine use on methadone maintenance treatment outcomes. J Addict Dis 2008; 27: 37-487

[66] Caplehorn J, Dalton M, Haldar F, et al. Methadone maintenance and addicts' risk of fatal heroin overdose. Subst Use Misuse 1996; 31: 177-196

[67] Mariani J, Malcolm R, Mamczur A et al. Pilot trial of gabapentin for the treatment of benzodiazepine abuse or dependence in methadone maintenance patients. Am J Drug Alcohol Abuse 2016; 6: 935-950

[68] Bleich A, Gelkopf, M, Weizman T, et al. Benzodiazepine abuse in a methadone maintenance treatment clinic in Israel: characteristics and a pharmacotherapeutic approach. Isr J Psychiatry Relat Sci 2002; 39: 104-112

[69] Gelkopf M, Bleich A, Hayward R, et al. Characteristics of benzodiazepine abuse in methadone maintenance patients: a 1 year prospective study in an Israeli clinic. Drug Alcohol Depend 1999; 55: 63-68
[70] Sharma V, Weir D, Samanani S, et al. Characterisation of Concurrent Use of Prescription Opioids and Benzodiazepine/Z-drugs in Alberta, Canada: A Population-Based Study. BMJ Open 2019; 9: e030858

[71] Stein M, Kanabar M, Anderson B, et al. Reasons for Benzodiazepine Use Among Persons Seeking Opioid Detoxification. J Subst Abuse Treat 2016; 68: 57-61

[72] Jones C, McAninch J. Emergency department visits and overdose deaths drom combined use of opioids and benzodiazepines. Am J Prev Med 2015; 49: 493-501

[73] Hernandez I, He M, Brooks M, et al. Exposure-Response Association Between Concurrent Opioid and Benzodiazepine Use and Risk of Opioid-Related Overdose in Medicare Part D Beneficiaries. JAMA Netw Open 2018; e180919

[74] MacLeod J, Steer C, Tilling K, et al. Prescription of Benzodiazepines, Z-Drugs, and Gabapentoids and Mortality Risk in People Receiving Opioid Agonist Treatment: Observational Study Based on the UK Clinical Practice Research Datalink and Office for National Statistics Death Records. Plos Med 2019; 26: e1002965

[75] Peles E, Schreiber S, Adelson M. 15-year survival and retention of patients in a general hospital-affiliated methadone maintenance treatment (MMT) center in Israel. Drug Alcohol Depend 2010; 107: 141-148

[76] Tori ME, Larochelle MR, Naimi TS. Alcohol or Benzodiazepine Co-Involvement with Opioid Overdose Deaths in the United States, 1999-2017. JAMA Network Open 2020; 3(4): e202361

[77] Cheng S, Siddiqui T, Gossop M, et al. Sociodemographic, Clinical and pharmacological Profiles of Medication Misuse and Dependence in Hospitalised Older Patients in Norway: A Prospective Cross-Sectional Study. BMJ Open 2019; 9: e031483

[78] Olfson M, King M, Schoenbaum M. Benzodiazepine use in the United States. JAMA Psychiatry 2015; 72: 136-42

[79] Sidorchuk A, Isomura K, Molero Y, et al. Benzodiazepine Prescribing for Children, Adolescents, and Young adults from 2006 Through 2013: A Total Population Register-Linkage Study. Plos Med 2018; e 1002635

[80] Hollister LE, Motzenbecker F, Degan R. Withdrawal reactions from chlordiazepoxide (“Librium”). Psychopharmacology 1961; 2: 63-8

[81] Inoue Y, Takaesu Y, Koebis M. Prevalence of and factors associated with acute withdrawal symptoms after 24 weeks of eszopiclone treatment in patients with chronic insomnia: a prospective, interventional study. BMC Psychiatry 2021; 21: 193

[82] Darker C, Sweeney B, Barry J, et al. Psychosocial interventions for benzodiazepine harmful use, abuse or dependence. Cochrane Database Syst Rev 2015; 5: CD009652

[83] Denis C, Fatseas M, Lavie E, et al. Pharmacological interventions for benzodiazepine mono-dependence management in outpatient settings. Cochrane Database Syst Rev 2006; CD005194

[84] Lingford-Hughes A, Welch S, Peters L, et al. BAP updated guidelines: evidence-based guidelines for the pharmacological management of substance abuse, harmful use, addiction and comorbidity: recommendations from BAP. J Psychopharmacol 2012; 26: 899-952

[85] Parr J, Kavanagh D, Cahill, L, et al. Effectiveness of current treatment approaches for benzodiazepine discontinuation: a meta-analysis. Addiction 2009; 104: 13-24

[86] Baillargeon L, Landreville P, Verrault R, et al. Discontinuation of Benzodiazepines among Older Insomniac adults treated 
with Cognitive-Behavioral Therapy Combined with Gradual Tapering: A Randomized Trial. CMAJ 2003; 169: 1015-1020

[87] Department of Health (England) and the devolved administrations. Drug Misuse and Dependence: UK Guidelines on Clinical Management. London: Department of Health (England), the Scottish Government, Welsh Assembly Government and Northern Ireland Executive; 2007

[88] Welsh JW, Tretyak V, McHugh RK, et al. Review: Adjunctive pharmacologic approaches for benzodaizepine tapers. Drug Alcohol Depend 2018; 189: 96-107

[89] Baandrup L, Ebdrup B, Rasmussen J, et al. Pharmacologic interventions for benzodiazepine users (Review). Cochrane Database Syst Rev 2018; 3: CD011481

[90] Paquin AM, Zimmerman K, Rudolph JL. Risk versus risk: a review of benzodiazepine reduction in older adults. Expert Opin Drug Safety 2014; 13: 919-934

[91] Welsh J, Tretyak V, McHugh K, et al. Review: Adjunctive pharmacologic approaches for benzodiazepine tapers. Drug Alcohol Depend 2018; 189: 96-107

[92] Nissen C, Frase I, Hajak G, et al. Hypnotika- Stand der Forschung. Nervenarzt 2014; 85: 67-76

[93] Morin CM, Bastien C, Guay B, et al. Randomized controlled trial of supervised tapering and cognitive-behavior therapy to facilitate benzodiazepine discontinuation in older adults with chronic insomnia. Am J Psychiatry 2004; 171: 332-342

[94] Everitt H, Baldwin D, Stuart B, et al. Antidepressants for insomnia in adults. Cochrane Database Syst Rev 2018; 14: 5 CD01753

[95] Sys J, van Cleynenbreugel S, Deschodt, M, et al. Efficacy and safety of non-benzodiazepine and non-Z-drug hypnotic medication for insomnia in older people: a systematic literature review. Eur J Clin Pharmacol 2020; 76: 363-381

[96] Cosci F, Chouiarnd G. Acute and Persistent Withdrawal Syndromes Following Discontinuation of Psychotropic Medications. Psychother Psychosom 2020. doi: 10.1159/00506868

[97] Nakao M, Takeuchi T, Nomura K, et al. Clinical Application of Paroxetine for Tapering Benzodiazepine Use in Non-Major Depressive Outpatients Visiting a Internal Medicine Clinic. Psychiatry Clin Neurosci 2006; 60: 605-610

[98] Zitman F, Couvée J. Chronic Benzodiazepine Use in General Practice Patients With Depression: An Evaluation of Controlled Treatment and Taper-Off: Report on Behalf of the Dutch Chronic Benzodiazepine Working Group. Br J Psychiatry 2001; 178: 317-324

[99] Schweizer E, Rickels K, Case W, et al. Carbamazepine treatment in patients discontinuing long-term, benzodiazepine therapy. Effects on withdrawal severity and outcome. Arch Gen Psychiatry 1991; 48: 448-452

[100] McHugh R, Votaw V, Bogunovic O, et al. Anxiety sensitivity and nonmedical benzodiazepine use among adults with opioid use disorder. Addict Behav 2017; 65: 283-288

[101] Olsen R. Gaba-A Receptor: Positive and Negative Allosteric Modulators. Neuropharmacology 2018; 136(pt A): 10-22

[102] Minozzi S, Saulle R. Rösner S. Baclofen for alcohol use disorder. Cochrane Database Syst Rev 2018; 11: CD012557

[103] Liu J, Lu-Ning W. Baclofen for alcohol withdrawal. Cochrane Database Syst Rev 2019. doi.wiley.com/10.1002/14651858. CD012557.pub2

[104] Shukla L, Kandasamy A, Kesevan M, et al. Baclofen in the short-term maintenance treatment of benzodiazepine dependence. J Neurosci in Rural Pract 2014; 5: S053-54

[105] Morena-Fumero A, Fernandez-Lopez L, Abreu-Gonzalez P. Melatonin and melatonin agonists as treatments for benzodiazepines and hypnotics in patients with primary insomnia. A systematic review. Drug Alcohol Depend 2020. doi.org/10.1016/j.drugalcdep2020.107994

[106] Bonnet U, Scherbaum N. How Addictive are Gabapentin and Pregabalin? A Systematic Review. Eur Neuropsychopharmacol 2017; 27: 1185-1215

[107] Caniff K, Telaga E, Bostwick, J, et al. Pregabalin as adjunctive therapy in benzodiazepine discontinuation. Am J Health-Syst Pharm 2018; 75: 67-71

[108] Cantopher T, Olivieri S, Cleave N, et al. Chronic benzodiazepine dependence. A comparative study of abrupt withdrawal under propranolol cover versus gradual withdrawal. $\mathrm{Br}$ J Psychatry 1990; 156: 406-411

[109] Rickels K, Schweizer E, Espana F, et al. Trazodone and Valproate in Patients Discontinuing Long-Term Benzodiazepine Therapy: Effects on Withdrawal Symptoms and Taper Outcome. Psychopharmacology 1999; 141: 1-5

[110] Janhsen K, Roser P, Hoffmann K. The Problems of Long-Term Treatment with Benzodiazepines and Related Substances. Dtsch Arztebl Int 2015; 112: 1-7

[111] Hood S, O'Neil G, Hulse G. The role of flumazenil in the treatment of benzodiazepine dependence: physiological and psychological profiles. J Psychopharmacol 2009; 23: 401-409

[112] Zhu S, Noviello C, Teng J, et al. Structure of human synaptic GABA-A receptor. Nature 2018; 559: 67-72

[113] Benini A, Gottardo R, Chiamulera C, et al. Continuous Infusion of Flumazenil in the Management of Benzodiazepines Detoxification. Front Psychiatry 2021. doi:10.3389/ psyt.2021. 646038

[114] Lugoboni F, Faccini M, Quaglio G, et al. Intravenous flumazenil infusion to treat benzodiazepine dependence should be performed in the clinical setting for high risk seizure. J Psychopharmacol 2011; 25: 848-849

[115] Wolter DK. Discontinuation of benzodiazepines in old age: When and if so, how? (in German). Gerontol Geriatr 2017; 50: $115-122$

[116] Mugunthan K, McGuire T, Glasziou P. Minimal Interventions to Decrease Long-Term Use of Benzodiazepines in primary Care. A Systematic Review and meta-Analysis. Br J Gen Pract 2011; 61: e573-578

[117] Heather N, Paton J, Ashton H. Predictors of response to brief intervention in general practice against long-term benzodiazepine use. Addiction Res Theory 2011; 19: 519-527

[118] Otto C, Crackau B, Lohrmann I, et al. Brief intervention in general hospital for problematic prescription drug use: 12-month outcome. Drug Alcohol Depend 2009; 105: 221-6

[119] Lynch T, Ryan C, Hughes C, et al. Brief interventions targeting long-term benzodiazepines and Z-drugs use in primary care: a systematic review and meta-analysis. Addiction 2020; 115: 1618-1639

[120] Merchant R, Baird J, Liu T. Short-term efficacy of a Brief Intervention to Reduce Drug Misuse and Increase Drug Treatment Utilization Among Adult Emergency Department Patients. Acad Emerg Med 2015; 22: 1172-1180

[121] Vicens C, Sempere E, Bejarano F, et al. Efficacy of Two interventions on the Discontinuation of Benzodiazepines in longTerm Users: 36-month Follow-Up of a Cluster randomised trial in Primary Care. Br J Gen Pract 2016; 66: e85-91

[122] de Gier N, Gorgels W, Lucassen J, et al. Discontinuation of long-Term Benzodiazepine Use: 10-year Follow-Up. Fam Pract 2011; 28: 253-259 
[123] Dutra L, Stathopoulou G, Basden S, et al. A meta-analytic review of psychosocial interventions for substance use disorders. Am J Psychiatry 2008; 165: 179-187

[124] Gould R, Coulson M, Patel N, et al. Interventions for Reducing Benzodiazepine Use in older people. Meta-Analysis of Randomised Controlled Trials. Br J Psychiatry 2014; 204: 98-107

[125] Royal College of General Practitioners. Prescribing Drugs of Dependence in General Practice, Part B - Benzodiazepines. Victoria: Royal College of General Practitioners; 2015

[126] Lader M, Kyriacou A. Withdrawing benzodiazepines in patients with anxiety disorders. Curr Psychiatry Rep 2016; 18: 8

[127] Dou C, Rebane J, Bardal S. Interventions to Improve Benzodiazepine Tapering Success in the Elderly: A Systematic Review. Aging Ment Health 2019; 23: 411-416

[128] Prochaska J, di Clemente C. (eds) Towards a comprehensive model of change. In. Miller W, Heather N (eds). Treating addictive behaviours. Process of change. New York: Plenum Press; 1986

[129] Miller WR, Rollnick S. Motivational interviewing: Preparing people for change. New York, NY: Guilford; 2002

[130] Ray L, Meredith L, Kiluk B, et al. Combined pharmacotherapy and Cognitive Behavioral Therapy for Adults with Alcohol or Substance Use Disorders. JAMA Network Open 2020; 3 : e208279

[131] Morgan K, Dixon S, Mathers N, et al. Psychological Treatment for insomnia in the Regulation of long-Term Hypnotic Drug Use. Health Technol Assess 2004; 8.iii-iv: 1-68

[132] Otto M, Pollack M, Sachs, G et al. Discontinuation of benzodiazepine treatment: efficacy of cognitive-behavioral therapy for patients with panic disorder. Am J Psychiatry 1993; 150: 1485-1490

[133] Reeve E, Ong M, Wu A, et al. A systematic Review of Interventions to Deprescribe Benzodiazepines and Other Hypnotics Among older People. J Clin Pharmacol 2017; 73 : 927-935

[134] Rickels K, Case W, Schweizer E, et al. Long-term Benzodiazepine Users 3 Years After Participation in a Discontinuation Program. Am J Psychiatry 1991; 148: 757-761

[135] Holton A, Riley P, Tyrer P. Factors predicting long-term outcome after chronic benzodiazepine therapy. J Affect Dis 1992; 24: $245-252$
[136] Oude Voshaar R, Gorgels W, Mol A, et al. Long-term outcome of two forms of randomised benzodiazepine discontinuation. Br J Psychiatry 2006; 188: 188-9

[137] Morin C, Bélanger L, Bastien C, et al. Long-term Outcome After Discontinuation of benzodiazepines for insomnia: a survival analysis of relapse. Behav Res Ther 2015; 43: 1-14

[138] Morin C, Culbert J, Schwartz S. Nonpharmacological interventions for insomnia: a meta-analysis of treatment efficacy. Am J Psychiatry 1994; 151: 1172-80

[139] Wolter DK. Sucht im Alter - Altern und Sucht. Stuttgart: Kohlhammer; 2010

[140] Gnjidic D, Ong H, Leung C, et al. The Impact of in Hospital Patient-Education Intervention on Older People's Attitudes and Intention to Have Their Benzodiazepines Prescribed: A Feasibility Study. Ther Adv Drug Saf 2019. doi.10.1177/2042098618816562

[141] Reeves R, Kamal A. Complicated Withdrawal Phenomena During Benzodiazepine Cessation in older Adults. J Am Osteopath 2019; 119: 327-331

[142] Baldwin D, Aitchison K, Bateson A, et al. Benzodiazepines: risks and benefits. A reconsideration. J Psychopharmacol 2013; 27: 967-71

[143] National Institute for Health and Clinical Excellence. Guidance on the Use of Zaleplon, Zolpidem and Zopiclone for Short-Term Management of Insomnia. London: National Institute for Health and Care Excellence; 2004

Bibliografie

Nervenheilkunde 2021; 40: 636-646

DOI $10.1055 / \mathrm{a}-1523-5718$

ISSN $0722-1541$

(c) 2021. Thieme. All rights reserved.

Georg Thieme Verlag KG, Rüdigerstraße 14,

70469 Stuttgart, Germany 


\section{Punkte sammeln auf CME.thieme.de}

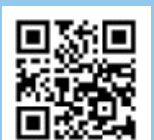

Diese Fortbildungseinheit ist bis zu 12 Monate online für die Teilnahme verfügbar.

Den genauen Einsendeschluss finden Sie beim Modul auf https://cme.thieme.de/CXHNNQG.

Sollten Sie Fragen zur Online-Teilnahme haben, finden Sie unter https://cme.thieme.de/hilfe eine ausführliche Anleitung. Wir wünschen viel Erfolg beim Beantworten der Fragen!

Unter https://eref.thieme.de/CXHNNQG oder über den QR-Code kommen Sie direkt zum Artikel.

VNR 2760512021160211185

\section{Frage 1}

Über welchen Transmitter wirken Benzodiazepine?
A Glutamat
B GABA
C Serotonin
D Noradrenalin
E Acetylcholin

\section{Frage 2}

Welcher Neurotransmitter im mesolimbischen System ist für das Suchtpotenzial von Substanzen entscheidend?
A Glutamat
B GABA
C Serotonin
D Dopamin
E Noradrenalin

\section{Frage 3}

Welches Benzodiazepinpräparat ist ein Anxiolytikum?
A Flurazepam
B Nitrazepam
C Temazepam
D Flunitrazepam
E Alprazolam

\section{Frage 4}

Zu den Hypnotika zählen ...
A Lorazepam.
B Alprazolam.
C Ozazepam.
D Midazolam.
E Bromazepam.

\section{Frage 5}

Zu den häufigen klinischen Problemen bei Benzodiazepinhypnotika gehören ...
A Hangover am nächsten Morgen.
B Leberwerterhöhungen.
C allergische Reaktionen.
D Haarausfall.
E Magenbeschwerden.

\section{Frage 6}

Zu dem NON-Benzodiazepinhypnotika zählen ...
A Gabapentin.
B Pregabalin.
C Z-Drugs.
D Baclofen.
E Promethazin.

\section{Frage 7}

Zu den häufigen Entzugserscheinungen bei abruptem Absetzen von Benzodiazepinen gehören ...
A epileptische Anfälle.
B Delir.
C Psychosen.
D Manie.
E Verwirrtheitszustände.

\section{Frage 8}

Weltweit liegt die Prävalenz für Missbrauch und Abhängigkeit von Benzodiazepinen etwa bei ...
A $0,5 \%$.
B $1 \%$.
C $2 \%$.
D $5 \%$.
E $10 \%$.

\section{Frage 9}

Ein sinnvoller Ansatz bei schrittweisem Absetzen von Benzodiazepinen bei Langzeitkonsum ist eine wöchentliche Reduktion um etwa ...
A $12,0 \%$.
B $25,0 \%$.
C $3,10 \%$.
D $4,25 \%$.
E $55,0 \%$.

\section{Frage 10}

Ein wichtiger, evidenzbasierter Therapieansatz in der Psychotherapie bei Benzodiazepinabhängigkeit ist ...
A kognitive Verhaltenstherapie.
B systemische Therapie.
C Psychoanalyse.
D Tiefenpsychologie.
E therapeutisches Reiten. 